\title{
A Joint Waveform and Precoding Design for Non-orthogonal Multicarrier Signals
}

\author{
Tongyang $\mathrm{Xu}$ and Izzat Darwazeh
}

\author{
Department of Electronic and Electrical Engineering, University College London, London, UK \\ Email: t.xu@ee.ucl.ac.uk, i.darwazeh@ucl.ac.uk
}

\begin{abstract}
In the spectrally efficient frequency division multiplexing (SEFDM) non-orthogonal multicarrier signal, higher spectral efficiency can be achieved at the expense of self-created inter carrier interference (ICI). The effective interference, which is contributed by all sub-carriers, has to be minimized and this results in a receiver of significant complexity. In order to mitigate the interference and simplify the receiver design, in this work, a precoding technique, based on eigenvalue decomposition of the sub-carrier correlation matrix, is utilised. Briefly, the technique is based on modifying the data sent on individual sub-carriers according to the signal quality of each, which is based on the sub-carrier to interference ratio (ScIR) of such sub-carrier as estimated from eigenvalue decomposition. A full system model is presented in this paper and simulations show that the precoding of SEFDM results in either better bit error rate (BER) performance compared to that of an orthogonal frequency division multiplexing (OFDM) system of the same spectral efficiency or in higher effective bit rate relative to an OFDM system with the same BER performance. Modelling is done in simple Gaussian noise channels and in a static frequency selective channel and for different modulation formats. Results show that for the same bandwidth a 128QAM precoded SEFDM system outperforms a 16QAM OFDM one by offering 75\% bit rate increase. Furthermore, Turbo coding assisted BER performance comparisons are investigated in this work. Using 64QAM modulated symbols, the precoded SEFDM outperforms the typical OFDM by several dBs.
\end{abstract}

\section{INTRODUCTION}

Multicarrier techniques are widely used in modern communication systems due to their high immunity against multipath fading. Orthogonal frequency division multiplexing (OFDM) [1] is the standardized technique in long term evolution (LTE) [2]. A key requirement of future $5^{t h}$ generation (5G) [3] networks is to support higher data rates in a limited spectrum, whilst using multicarrier signal formats. A potential solution, termed spectrally efficient frequency division multiplexing (SEFDM) [4], compresses occupied signal bandwidth by packing sub-carriers at frequency spacing below the symbol rate. Thus, the spectral efficiency is higher than that of OFDM. However, the bandwidth is compressed at the cost of increased interference between the sub-carriers and therefore increased receiver complexity.

Many researchers have investigated optimal signal detection algorithms conceptually and practically. The history of SEFDM signal detection algorithms development is detailed in [5]. To summarize, the existing detectors have trade-off issues in performance, complexity and spectral efficiency.
Some detectors show optimal performance but with high complexity. Some detectors have low complexity but at the cost of performance. While some detectors achieve good performance and low complexity but with reduced achievable spectral efficiency. Therefore, the topic of efficient signal detection still remains open. Existing SEFDM detectors attempt to extract useful information from distorted signals at the receiver, which would result in inaccurate signal estimate since inter carrier interference (ICI) has been added to each sub-carrier. An alternative solution is to precode signals prior to the wireless channel at the transmitter, based on known ICI information. The precoding makes use of eigenvalue decomposition of [6], which would redistribute the power on each sub-carrier after symbol demodulation at the receiver. Depending on the percentage of bandwidth compression, some sub-carriers would experience power enhancement while others would experience power attenuation. The original precoding work in [6] aimed to transmit data on both strong (i.e. high sub-carrier to interference ratio (ScIR)) sub-carriers and weak (i.e. low ScIR) sub-carriers. As mentioned in [6], simple detectors such as zero forcing (ZF) can be applied for the strong channels while high performance detectors such as maximum likelihood (ML) are used for the weak channels. However, this approach requires the increase of complexity at the receiver, which does not satisfy the initial purpose of precoding. It should be noted that the approach of [6] is only applicable to small size systems. With the increase of system size, the detection for the weak channels becomes exceptionally complicated.

This work concentrates on the simplification of the precoding scheme, in a static frequency selective channel. A signal model, which occupies the same bandwidth, is tested for the precoded SEFDM without complicated detection algorithms. Results show that due to the bandwidth compression benefit, SEFDM is able to offer constructive power distribution to a portion of sub-carriers, which indicates an improved performance on those sub-carriers. In a frequency selective channel, both uncoded and Turbo coded simulation results show that SEFDM signals outperform OFDM signals. This is due to the benefit of frequency diversity that specially exists in the precoded SEFDM.

\section{BANDWIDTH COMPRESSED WAVEFORM}

The sub-carriers packing scheme of SEFDM is illustrated in Fig. 1. It is evident that the SEFDM waveform packs sub- 


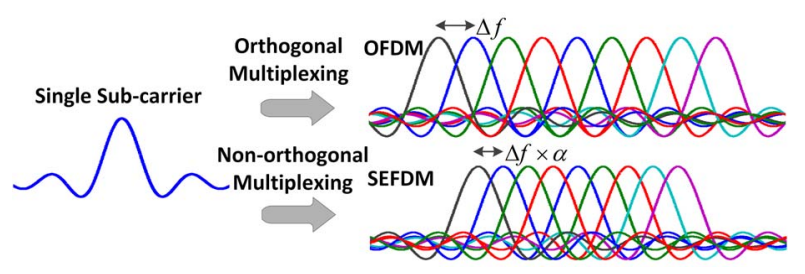

Figure 1. Waveform comparisons of OFDM and SEFDM.

carriers closer, leading to bandwidth saving at the cost of selfcreated ICI. The waveforms from Fig. 1 can be uniformly expressed mathematically in (1) where flexible choice of $\alpha$ determines the signal bandwidth compression.

$$
X[k]=\frac{1}{\sqrt{Q}} \sum_{n=0}^{N-1} s_{n} \exp \left(\frac{j 2 \pi n k \alpha}{Q}\right),
$$

where the bandwidth compression factor $\alpha=\Delta f T, \Delta f$ is sub-carrier spacing and $T$ is the period of one SEFDM symbol. $Q=\rho N$ is the number of samples for one SEFDM symbol and $\rho \geq 1$ is oversampling factors. $\frac{1}{\sqrt{Q}}$ is a normalization factor, $s_{n}$ is the QAM symbol modulated on the $n^{t h}$ sub-carrier and $X[k]$ is the $k^{t h}$ time sample with index $k=[0,1, \ldots, Q-1]$. A simplified expression of (1) in matrix form is

$$
X=\mathbf{F} S
$$

where $\mathbf{F}$ is a $Q \times N$ sub-carrier matrix with elements equal to $\frac{1}{\sqrt{Q}} e^{\frac{j 2 \pi n k \alpha}{Q}}$ and $S$ is a vector consisting of $N$ M-QAM symbols. The $N \times N$ correlation matrix, which offers ICI information, is expressed as

$$
\mathbf{C}=\mathbf{F}^{*} \mathbf{F}
$$

where $\mathbf{F}^{*}$ is the conjugate sub-carriers matrix.

\section{PRECODING}

Precoding concept can make use of ICI information at the transmitter and simplify the design of the receiver. The correlation matrix $\mathbf{C}$ characterizes the interference among the sub-carriers. The matrix can be diagonalized using eigenvalue decomposition [7] as

$$
\mathbf{C}=\mathbf{U} \Lambda \mathbf{U}^{*}
$$

where $\mathbf{U}$ is a unitary matrix and $\mathbf{U}^{*}$ is its conjugate with the property as $\mathbf{U}^{*} \mathbf{U}=\mathbf{I}$ where $\mathbf{I}$ is an identity matrix and $\boldsymbol{\Lambda}$ is a diagonal matrix that contains the eigenvalues $\operatorname{diagonal}(\boldsymbol{\Lambda})=$ $\left[\lambda_{1}, \lambda_{2}, \ldots, \lambda_{N}\right]$ of $\mathbf{C}$.

The eigenvalues, calculated according to (4), are illustrated in Fig. 2 where OFDM and SEFDM systems are compared. It is noted that for an OFDM system with $N$ sub-carriers, all eigenvalues have equal amplitude, which is equal to 'one'. For SEFDM systems, $\alpha N$ of the eigenvalues will have values greater than 'one' while the remaining $(1-\alpha) N$ eigenvalues can take very small values. It can be inferred that SEFDM detection systems that rely on matrix inversion will result in poor BER performance due to those small eigenvalues.

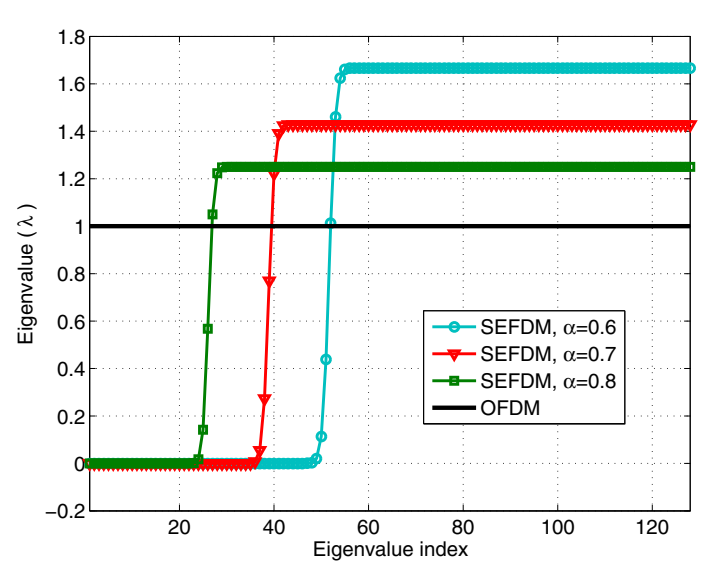

Figure 2. Eigenvalue versus eigenvalue index. A total number of $\mathrm{N}=128$ sub-carriers are tested.

The main idea of the precoding system, discussed here, is to recover a signal vector in which signal power is redistributed according to the eigenvalues. Small eigenvalues, therefore low ScIR, indicate weak channels and high eigenvalues, therefore high ScIR, indicate strong channels. Therefore, the precoding method leads to high performance for the strong channels while it lowers the performance for the weak channels.

The original transmitted symbols vector $S$ is multiplied by the unitary matrix U. Thus, the precoded symbols vector $\bar{S}$ is expressed as

$$
\bar{S}=\mathbf{U} S
$$

where the power of the precoded symbols vector is calculated as

$$
\|\bar{S}\|^{2}=S^{*} \mathbf{U}^{*} \mathbf{U} S=\|S\|^{2}
$$

showing that the total power of the transmitted symbols is maintained after multiplying with a unitary matrix U. Then, the precoded symbols are modulated using IFFT [8] as

$$
X=\mathbf{F} \bar{S}
$$

After transmitting $X$ through an additive white Gaussian noise (AWGN) channel, denoted by $\mathrm{Z}$, the received signal is expressed as

$$
Y=X+Z
$$

After demodulation using an FFT [8], the collected statistic vector $R$ is derived as

$$
R=\mathbf{F}^{*}(X+Z)=\mathbf{F}^{*} \mathbf{F} \bar{S}+\mathbf{F}^{*} Z=\mathbf{C} \bar{S}+Z_{\mathbf{F}^{*}}
$$

where $R$ is an $N$-dimensional vector of demodulated symbols, $Z_{\mathrm{F}^{*}}$ is the AWGN correlated with the conjugate sub-carriers. 


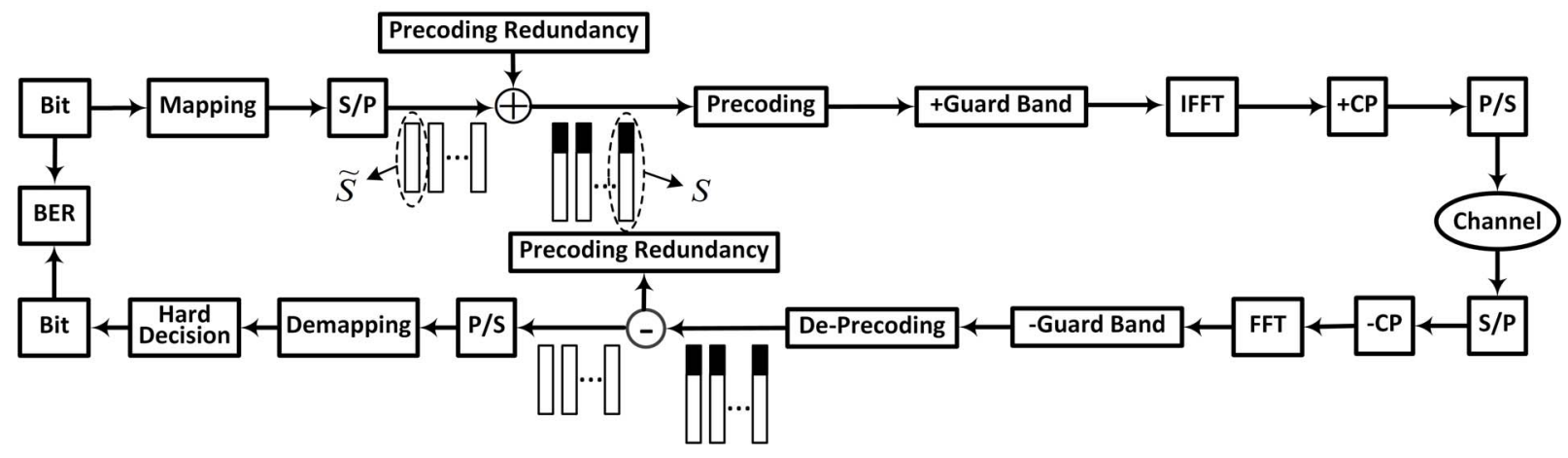

Figure 3. Simulation block diagram of the precoding scheme of SEFDM.

Removal of precoding is done in a de-precoding stage, which is effected by left multiplying (9) with $\mathbf{U}^{*}$, a new vector $\bar{R}=\left[\bar{r}_{1}, \bar{r}_{2}, \ldots, \bar{r}_{N}\right]$ is obtained as

$$
\begin{aligned}
\bar{R} & =\mathbf{U}^{*} R \\
& =\mathbf{U}^{*} \mathbf{C} \bar{S}+\mathbf{U}^{*} Z_{\mathbf{F}^{*}} \\
& =\Lambda \mathbf{U}^{*} \bar{S}+Z_{\mathbf{U}^{*}} \\
& =\Lambda S+Z_{\mathbf{U}^{*}}
\end{aligned}
$$

Equation (10) indicates that the power is re-distributed on each M-QAM symbols $S$ based on the eigenvalues $\operatorname{diagonal}(\boldsymbol{\Lambda})=\left[\lambda_{1}, \lambda_{2}, \ldots, \lambda_{N}\right]$. Then vector scaling is operated as

$$
\hat{s}_{i}=\bar{r}_{i} / \lambda_{i}
$$

where $i \in[(1-\alpha) \times N+1, N]$. It is inferred that the scaling operation in (11) would enchance the noise effect on the sub-carriers associated with small eigenvalues while it lowers the noise effect on the sub-carriers associated with high eigenvalues. Thus, the first $(1-\alpha) \times N$ sub-carriers result in low performance while the rest $\alpha \times N$ sub-carriers lead to better performance. In this work, the $\alpha \times N$ sub-carriers carry user data while redundant data for precoding is modulated on the $(1-\alpha) \times N$ sub-carriers.

Since a portion of sub-carriers are effectively used for user data, symbol rate is reduced by a factor of $(1-\alpha)$, but since the bandwidth is compressed by a factor of $(1-\alpha)$, the spectral efficiency is not changed. In order to have a fair comparison where equal occupied bandwidth is used for SEFDM and OFDM, a higher number of sub-carriers can be aggregated in the SEFDM system. Thus the symbol rate will be improved.

\section{Design and Simulations}

The simulation setup of the joint waveform and precoding system is shown in Fig. 3. In this section, signals are tested over the same bandwidth. Therefore, the total number of sub-carriers is $\frac{N}{\alpha}$, which is inversely proportional to the bandwidth compression factor. At the transmitter, a bit stream is mapped to a symbol stream in the mapping block based on a specific modulation format (e.g. M-QAM). After serialto-parallel (S/P) conversion, multiple symbol blocks $\tilde{S}$ are obtained with each consisting of $N$ M-QAM symbols. Then, $\left(\frac{1}{\alpha}-1\right) \times N$ precoding redundant M-QAM symbols are inserted at the beginning of each symbol block $\tilde{S}$ leading to multiple symbol blocks $S$ in which $\frac{N}{\alpha}$ M-QAM symbols are aggregated. The precoding follows the operations described in section III. A guard band is added on both sides of each precoded symbol before the IFFT [8] for oversampling and protection purposes. The modulated signal, after cyclic prefix (CP) addition, is then parallel-to-serial (P/S) converted and finally delivered through a channel.

At the receiver, the $\mathrm{S} / \mathrm{P}$ converted signal is $\mathrm{CP}$ removed and demodulated using a specially designed FFT algorithm [8] followed by the guard band removal. The de-precoding follows the operations in section III where matrix multiplication and vector scaling operation are required. The precoding redundancy is removed to recover the information data. After $\mathrm{P} / \mathrm{S}$ conversion, demapping and hard decision processing, the receiver bit stream is recovered. Finally, BER will be calculated comparing the transmitter bit stream and the receiver bit stream.

Table I

Simulation SySTEM SPECIFICATIONS

\begin{tabular}{lll}
\hline Parameters & OFDM & SEFDM \\
\hline \hline Sampling rate $(\mathrm{MHz})$ & 30.72 & 30.72 \\
\hline Bandwidth $(\mathrm{MHz})$ & 18 & 18 \\
\hline FFT size & 2048 & 2048 \\
\hline CP length & 144 & 144 \\
\hline Sub-carrier bandwidth $(\mathrm{kHz})$ & 15 & 15 \\
\hline Symbol rate per sub-carrier $(\mathrm{kS} / \mathrm{s})$ & 15 & 15 \\
\hline Sub-carrier spacing $(\mathrm{kHz})$ & 15 & $15 \times \alpha$ \\
\hline Bandwidth compression factor $\alpha$ & 1 & $0.8,0.7,0.6$ \\
\hline Guard band sub-carriers & 848 & $548,334,48$ \\
\hline Number of data sub-carriers & 1200 & $1500,1714,2000$ \\
\hline Modulation scheme (M-QAM) & $64,16,4$ & $128,64,32,16,8,4$ \\
\hline Precoding redundancy & 0 & $300,514,800$ \\
\hline Effective symbol rate $(\mathrm{MS} / \mathrm{s})$ & 18 & 18 \\
\hline
\end{tabular}

The simulation specifications for both OFDM and precoded SEFDM systems are shown in Table I. In this work, SEFDM systems are designed to be compatible with existing LTE OFDM systems [2]. By reconfiguring the guard band subcarriers length (e.g. 548 for $\alpha=0.8,334$ for $\alpha=0.7$ and 48 for $\alpha=0.6$ ), three SEFDM systems of equal bandwidth are 
studied. LTE defined modulation formats such as 64QAM, 16QAM and 4QAM are tested for OFDM systems. For the purpose of fair and sufficient comparisons, various modulation formats ranging from 4QAM to 128QAM are evaluated for SEFDM systems. It should be noted that in SEFDM systems, data sub-carriers and precoding redundant sub-carriers are modulated using the same modulation schemes for the purpose of simplicity.

\section{A. AWGN Scenario}

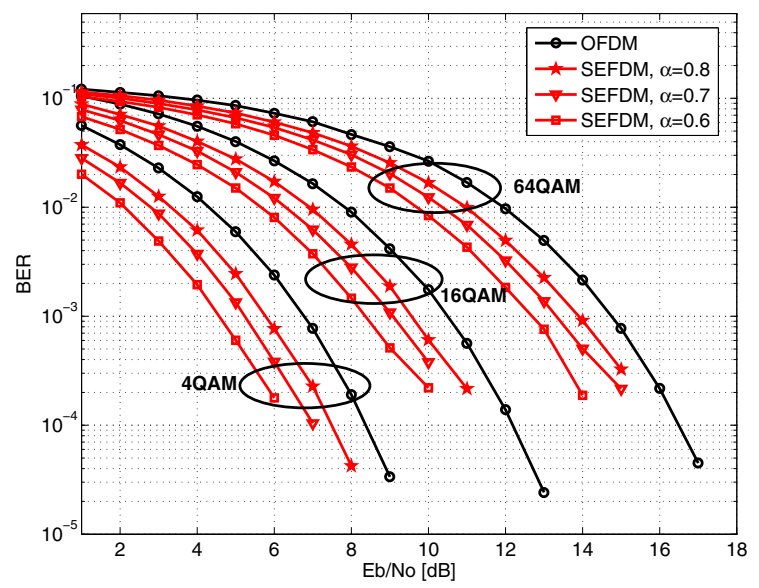

Figure 4. BER comparisons in AWGN channel under various bandwidth compression factors.

The BER comparisons of the conventional OFDM and the precoded SEFDM are investigated by considering Fig. 4, where the effect of different bandwidth compression factors of the precoded SEFDM is evaluated. For $\alpha=0.8$, the BER performance is approximately $1 \mathrm{~dB}$ better than the conventional OFDM, for all the modulation formats studied. Reducing the bandwidth compression factor $\alpha$ to 0.7 , the BER curve moves away from the OFDM one with $0.5 \mathrm{~dB}$ improvement. With further reduction of $\alpha$ to 0.6 , the performance is further improved and $2 \mathrm{~dB}$ gain is achieved compared with the OFDM result. It is concluded that by using the same modulation format, therefore the same spectral efficiency, the precoded SEFDM outperforms OFDM with flexible performance gains, which are related to the values of bandwidth compression factor $\alpha$. This counterintuitive result of improvement of the error performance with reduction of alpha is due to the fact that lower values of alpha require higher levels of precoding and therefore result in better performance at the expense of larger FFT sizes and smaller guard band sub-carriers. Finding an optimum value of alpha is the subject of current work.

The benefit of the precoded SEFDM in terms of data rate improvement is presented in Fig. 5 where three additional modulation formats; 128QAM, 32QAM and 8QAM are tested. It is apparent that the 8QAM modulated SEFDM signal has close performance to that of the 4QAM modulated OFDM signal. Since each of the 8QAM symbol includes 3 bits and

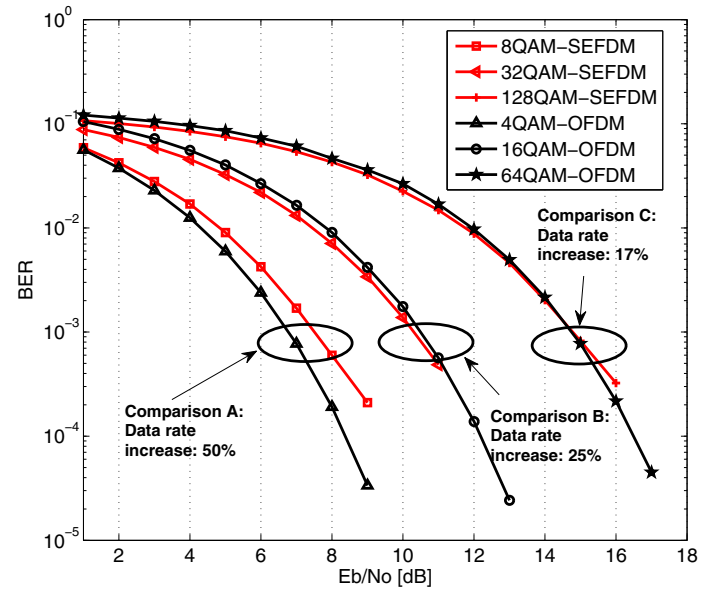

Figure 5. Bit rate comparisons of $\alpha=0.6$ precoded SEFDM and OFDM in the condition of various modulation formats.

each 4QAM symbol consists of 2 bits, therefore, the data rate is improved by $50 \%=(3 / 2-1) \times 100 \%$. With the increase of modulation format order, the data rate improvement is still evident in SEFDM but with reduced benefits. For example, comparing the 32QAM modulated SEFDM signal and the 16QAM modulated OFDM signal, the data rate is increased by $25 \%=(5 / 4-1) \times 100 \%$. Furthermore, with higher modulation formats such as 128QAM modulated SEFDM signal and 64QAM modulated OFDM signal, the percentage is reduced to around $17 \%=(7 / 6-1) \times 100 \%$. To sum up, the tested precoded SEFDM system can achieve the same performance compared to an OFDM system while achieving increased data rates, which are jointly set by modulation formats and bandwidth compression factors.

\section{B. Multipath Propagation Scenario}

In this section, a perfect channel state information (CSI) is assumed at the receivers for both OFDM and SEFDM in order to mitigate the effect of channel estimation. For detailed description of a time-domain channel estimation algorithm of SEFDM, readers are referred to the work in [9]. This section will briefly present the principle of the time-domain channel equalization for SEFDM and frequency-domain channel equalization for OFDM.

Considering a joint multipath and AWGN channel, the receiver side distorted signal after CP removal [9] is expressed as

$$
Y_{c}=\mathbf{H}_{\mathbf{c}} X+Z=\mathbf{H}_{\mathbf{c}} \mathbf{F} S+Z
$$

where $Y_{c}$ is a vector of length $Q$. The channel $\mathbf{H}_{\mathbf{c}}$ is a $Q \times$ $Q$ circulant matrix, which is a truncated (i.e. removing $\mathrm{CP}$ effect) version of a channel Toeplitz matrix $\mathbf{H}$ shown in [9]. After demodulation, which is effectively a multiplication of $Y_{c}$ with the conjugate sub-carrier matrix $\mathbf{F}^{*}$, the signal vector is expressed as

$$
R=\mathbf{F}^{*} \mathbf{H}_{\mathbf{c}} \mathbf{F} S+\mathbf{F}^{*} Z=\mathbf{G} S+Z_{\mathbf{F}^{*}} .
$$


For orthogonal multicarrier signals (e.g. OFDM), $\mathbf{G}$ is a diagonal matrix. Thus, the channel can be estimated and compensated through a one tap frequency-domain equalizer. However, this is not the case in SEFDM since there are off-diagonal elements in the matrix $\mathbf{G}$. This introduces both multiplicative (diagonal elements) and additive (off-diagonal elements) distortions. Therefore, OFDM and SEFDM have different solutions to compensate for channel effects.

For OFDM, channel equalization with perfect CSI is operated as

$$
\hat{s}_{i}=r_{i} / g_{i}, i=0,1, \ldots, N-1
$$

where $\hat{s}_{i}$ is the $i^{t h}$ element of estimate $\hat{S}, r_{i}$ is the $i^{t h}$ element of $R$ and $g_{i}$ is the $i^{\text {th }}$ element of $\operatorname{diagonal}(\mathbf{G})$.

For SEFDM, the known channel matrix $\mathbf{H}_{\mathbf{c}}$ is inverted to give $\mathbf{H}_{\mathbf{c}}^{-1}$ and used to equalize the distorted SEFDM symbols in time domain in (12) as

$$
\begin{aligned}
Y_{e q}=\mathbf{H}_{\mathbf{c}}^{-1} Y_{c} & =\mathbf{H}_{\mathbf{c}}^{-1} \mathbf{H}_{\mathbf{c}} \mathbf{F} S+\mathbf{H}_{\mathbf{c}}^{-1} Z \\
& =\mathbf{F} S+Z_{\mathbf{H}_{\mathbf{c}}^{-1}}
\end{aligned}
$$

Then, following the operations in (9), (10) and (11), SEFDM symbols will be recovered.

To test this, a static frequency selective channel, obtained from [10], is used in the simulation as

$$
\begin{array}{r}
h(t)=0.8765 \delta(t)-0.2279 \delta\left(t-T_{s}\right)+0.1315 \delta\left(t-4 T_{s}\right) \\
-0.4032 e^{\frac{j \pi}{2}} \delta\left(t-7 T_{s}\right)(16)
\end{array}
$$

where $T_{s}$ is the sampling period, which can be calculated according to the system specifications in Table I. The spectra of OFDM and precoded SEFDM signals distorted by the static channel are shown in Fig. 6 where the occupied bandwidth is $18 \mathrm{MHz}$ for both cases. Although both spectra have the same frequency response, the OFDM spectrum aggregates $N$ subcarriers while the SEFDM one aggregates $\frac{N}{\alpha}$ sub-carriers.

For the OFDM signals, after symbol mapping, each symbol is modulated on a separate sub-carrier. In the precoded SEFDM signals, after symbol mapping, the precoding operation will spread each symbol over multiple sub-carriers, which indicates multiple sub-carriers will carry one symbol. Thus, in a frequency selective channel, the precoded SEFDM will offer frequency diversity.

In order to fully explore the benefits of precoded SEFDM in the static frequency selective channel, various modulation formats ranging from 4QAM to 128QAM are tested with results showing in Fig. 7. For the same modulation formats, it is clear that the error performance of precoded SEFDM signals shows several $\mathrm{dBs}$ of advantage compared to that of the OFDM signals. When a precoded SEFDM signal is used, applying a higher modulation format leads to almost identical error performance at low $E b / N o$ and much better performance at high $E b / N o$. This is due to the effect of precoding leading to BER curves slope difference where the slope of the SEFDM curves is greater than that of the OFDM curves. One achievement is the comparison of 128QAM SEFDM and 16QAM OFDM. It is noted that below $E b / N_{O}=19 \mathrm{~dB}$, the

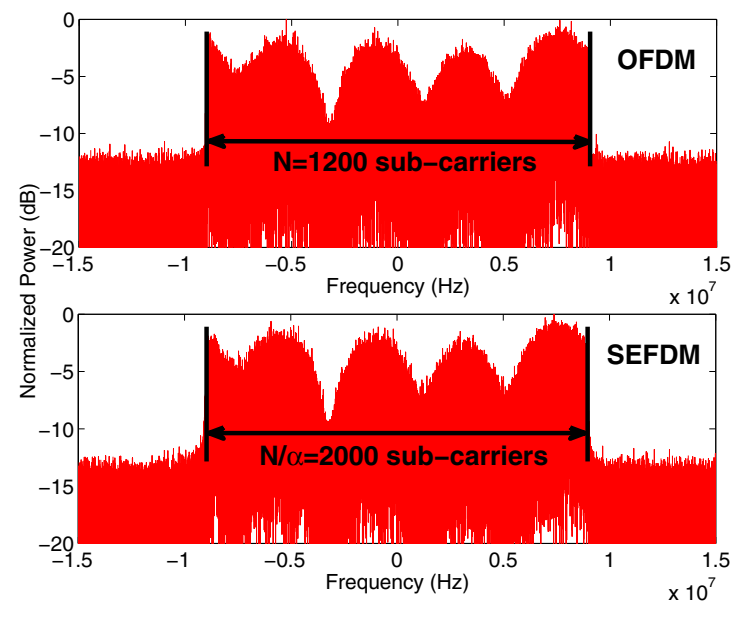

Figure 6. Spectra of OFDM and $\alpha=0.6$ SEFDM signals after the static frequency selective channel. Occupying the same bandwidth, a total number of $N=1200$ data sub-carriers are aggregated for OFDM and $N / \alpha=2000$ data sub-carriers for SEFDM.

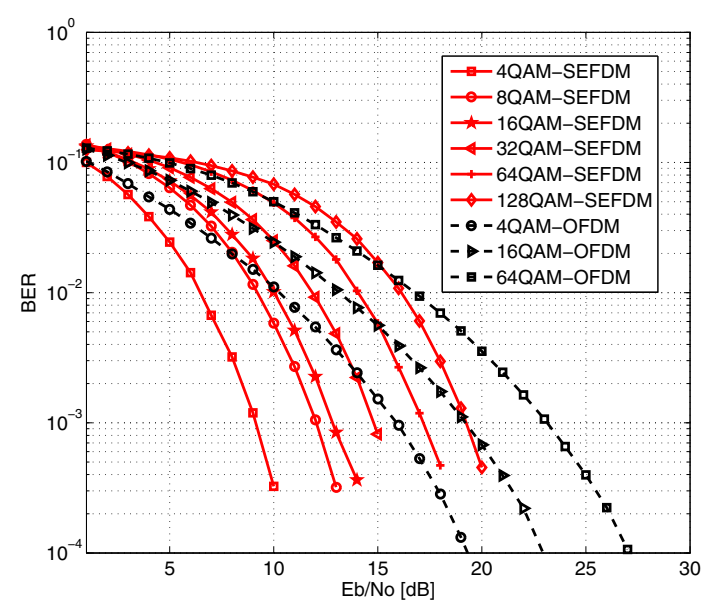

Figure 7. BER comparisons of $\alpha=0.6$ precoded SEFDM signals and OFDM signals in a static frequency selective channel.

OFDM system outperforms the SEFDM system while beyond $E b / N o=19 \mathrm{~dB}$, the 128QAM SEFDM starts performing better than the 16QAM OFDM one.

The reason for such performance improvement is attributed to the fact that this joint compressed waveform and precoding scheme provides frequency diversity in frequency selective channels, since not only channel effects are spread over all sub-carriers, but also only a portion (i.e. $(100 \times \alpha) \%)$ of subcarriers carry information data and these are the ones benefitting most from the precoding and eigenvalue decomposition process.

The precoding technique can achieve better performance since it can exploit the frequency diversity. In realistic communication systems, Turbo error correcting codes are commonly used due to their powerful error correction capability over 


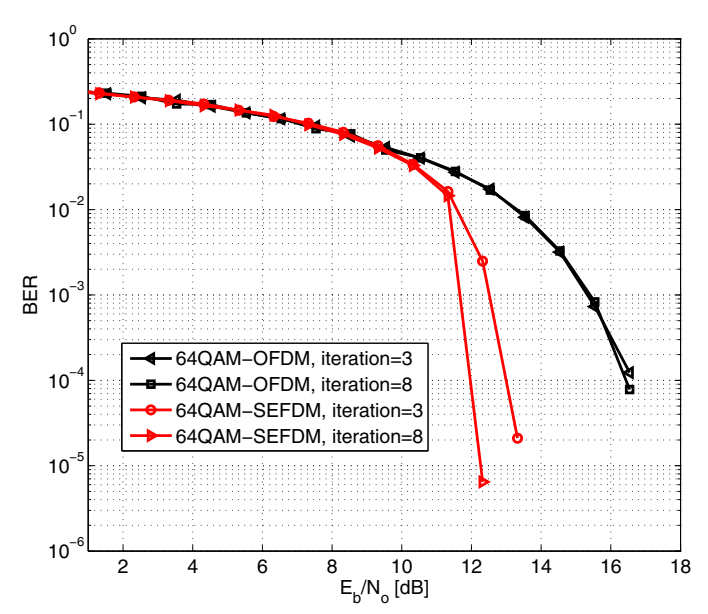

Figure 8. Turbo coded BER comparisons of $\alpha=0.6$ precoded SEFDM signals and OFDM signals in a static frequency selective channel.

multipath channels. Fig. 8 further explores performance in a Turbo coding scenario to verify that the precoding technique can still have advantages when used in conjunction with Turbo coding. For the simulation, a rate $1 / 3$ parallel concatenated Turbo code with polynomials $G_{1}(D)=1+D^{2}+D^{3}$ and $G_{2}(D)=1+D+D^{3}$, derived from LTE [11], are used for both cases; the typical OFDM and precoded SEFDM. Both systems are tested in the same frequency selective channel scenario used to obtain the results of the uncoded systems of Fig. 7. The 64QAM modulated OFDM signals with three and eight iterations are evaluated. It is evident that both have the same error performance. It indicates that three iterations are sufficient to achieve optimal BER performance and increasing the number of iterations will not improve performance. Meanwhile, the precoded SEFDM signal with the same modulation scheme is tested using also three and eight iterations. The two tested SEFDM cases perform better than the typical OFDM cases by several dBs. It is inferred from the above simulations that the use of Turbo coding can improve the performance of both systems and the performance gain achieved by the precoded SEFDM signal is still maintained.

\section{Complexity}

The non-orthogonal nature of SEFDM necessitates additional computational effort. Standard transmitter IFFT can not be used, however work in [8] introduced efficient signal modulation algorithms with greatly reduced complexity. Additional computational effort is also needed for the precoding operation, shown in (4), which requires eigenvalue decomposition of a $N \times N$ matrix. This work assumes perfect CSI and does not consider channel estimation at the receiver. However, it should be noted that in realistic systems channel estimation adds significantly to the computational effort relative to typical OFDM estimation methods since the channel matrix $\mathbf{H}_{\mathbf{c}}$, shown in (12), has to be estimated in SEFDM whereas in OFDM one-tap frequency domain equalization is used. More detailed treatment can be found in [12]. In addition, channel equalization in (15) requires the inversion operation of the matrix $\mathbf{H}_{\mathbf{c}}$. In general, the benefits of SEFDM, come at the cost of extra complexity, mainly at the receiver [13].

\section{CONCLUSions}

A joint bandwidth compressed and precoded scheme was studied and verified in this work. Eigenvalue decomposition was utilized to simplify the receiver design. In AWGN channel, for the same condition of modulation format and occupied bandwidth, the precoded SEFDM systems outperform its OFDM equivalent. In a frequency selective channel, the precoding introduces frequency diversity since one symbol will be modulated on multiple sub-carriers. Thus, the precoded SEFDM would be more robust in frequency selective channels. Simulations show, with the help of error correcting codes, a precoded SEFDM signal modulated by 128QAM symbols outperforms a 16QAM modulated OFDM signal in multipath channel scenarios. In addition, the precoded SEFDM and the typical OFDM systems, both modulated by 64QAM symbols, are tested in the predefined frequency selective channel. Results indicate that the power advantage of the precoded SEFDM is maintained over OFDM.

\section{REFERENCES}

11] T. Hwang, C. Yang, G. Wu, S. Li, and G. Y. Li, "OFDM and its wireless applications: A survey," IEEE Transactions on Vehicular Technology, vol. 58, no. 4, pp. 1673-1694, May 2009.

[2] 3GPP TS 36.300 version 8.12.0 Release 8, "Evolved universal terrestrial radio access (E-UTRA) and evolved universal terrestrial radio access network (E-UTRAN); overall description; stage 2 (release 8)," April 2010.

[3] J. Andrews, S. Buzzi, W. Choi, S. Hanly, A. Lozano, A. Soong, and J. Zhang, "What will $5 \mathrm{G}$ be?" Selected Areas in Communications, IEEE Journal on, vol. 32, no. 6, pp. 1065-1082, June 2014.

[4] M. Rodrigues and I. Darwazeh, "A spectrally efficient frequency division multiplexing based communications system," in Proc. 8th Int. OFDM Workshop, Hamburg, 2003, pp. 48-49.

[5] F.-L. Luo and C. J. Zhang, Signal Processing for 5G: Algorithms and Implementations. Wiley, 2016.

[6] S. Isam and I. Darwazeh, "Precoded spectrally efficient FDM system," in Personal Indoor and Mobile Radio Communications (PIMRC), 2010 IEEE 21st International Symposium on, Sept 2010, pp. 99-104.

[7] G. Strang, Introduction to linear algebra. Wellesley-Cambridge Press, 2009.

[8] P. Whatmough, M. Perrett, S. Isam, and I. Darwazeh, "VLSI architecture for a reconfigurable spectrally efficient FDM baseband transmitter," Circuits and Systems I: Regular Papers, IEEE Transactions on, vol. 59, no. 5, pp. 1107-1118, May 2012.

[9] T. Xu, S. Mikroulis, J. E. Mitchell, and I. Darwazeh, "Bandwidth compressed waveform for $60-\mathrm{GHz}$ millimeter-wave radio over fiber experiment," Journal of Lightwave Technology, vol. 34, no. 14, pp. 3458-3465, July 2016.

[10] X. Wang, P. Ho, and Y. Wu, "Robust channel estimation and ISI cancellation for OFDM systems with suppressed features," Selected Areas in Communications, IEEE Journal on, vol. 23, no. 5, pp. 963-972, May 2005.

[11] 3GPP TS 36.212 v.12.2.0, "Evolved universal terrestrial radio access (E-UTRA); multiplexing and channel coding," Rel. 12, Oct. 2014

[12] S. Isam and I. Darwazeh, "Robust channel estimation for spectrally efficient FDM system," in Telecommunications (ICT), 2012 19th International Conference on, April 2012, pp. 1-6.

[13] I. Kanaras, A. Chorti, M. Rodrigues, and I. Darwazeh, "Spectrally efficient FDM signals: Bandwidth gain at the expense of receiver complexity," in Communications, 2009. ICC '09. IEEE International Conference on, June 2009, pp. 1-6. 